\title{
Small-size Coaxial Resonant Applicator for Microwave Heating Assisted Additive Manufacturing
}

\author{
Nanya Li, Guido Link, Moritz Engler, and John Jelonnek, Senior Member, IEEE
}

\begin{abstract}
This paper introduces the design and analysis of a small-size coaxial resonant applicator for high-speed microwave heating assisted additive manufacturing of multiple materials, such as continuous carbon fiber reinforced polymer composites, thermoplastic and metal parts. The elaborated coaxial resonant applicator reduced the size and has a resonant frequency between 2.4 to $2.5 \mathrm{GHz}$. A TEM wave is stimulated in the applicator where the electrical field is polarized perpendicular to the filaments and therefore allows a maximum penetration depth. The electrical conductive filament is designed as part of the inner conductor to enhance coupling efficiency. To prevent microwave leakage induced by the conductive material, a compact quarter wavelength filter was developed. The equivalent circuit of the filter was used to analyze the influence of structural parameters on the resonance frequency. The filter has been tested and good agreement between measured and simulated results is obtained. The heating behaviour with varying input power has been investigated for polyamide, polylactic acid and continuous carbon fiber reinforced polyamide filaments.
\end{abstract}

Index Terms - tunable resonators, microwave filters, composite material structures, simulation and modeling, cavities.

\section{INTRODUCTION}

$\mathrm{M}$ ICROWAVE additive manufacturing of continuous carbon fiber reinforced thermoplastics (CFRTP) with high-speed has been presented by the authors recently [1]. The first prototype of microwave heating assisted additive manufacturing system has been developed to print highperformance CFRTP parts. The unique system can provide over 10 times printing speed of continuous fiber reinforced thermoplastic materials using the conventional hot end $[2,3]$. Currently, similar to the commercial filaments [4], a CFRTP filament produced by the authors has about $0.45 \mathrm{~mm}$ diameters ( $1 \mathrm{~K}$ carbon fibers, $23 \% \sim 25 \%$ carbon fiber volume fraction). The selective and volumetric microwave heating of filaments with a larger diameter $6 \mathrm{~K}$ carbon fiber, $1.05 \mathrm{~mm}$ diameter, 27\% fiber volume fraction) [5] offers the possibility

This paper has been submitted on June 152021 for review. This work was support by the Young Investigator Group Preparation Program (YIG Prep Pro) at Karlsruhe Institute of Technology (KIT), Alexander von Humboldt Foundation as well as to the donor, the Federal Ministry for Education and Research. (Corresponding author: Nanya Li)

Nanya Li, Guido Link, Moritz Engler and John Jelonnek are with the Institute for Pulsed Power and Microwave Technology, KIT, EggensteinLeopoldshafen, 76344, Germany (e-mail: nanya.li@kit.edu). of printing large-scale CFRTP structures. However, the conductive carbon fibers can induce the leakage of microwaves, and an efficient filter is needed. Next, to provide reliable temperature control, the electromagnetic field needs to be focused onto a small area of the filament and can operate multiple materials. Hence, based on these preliminary works, a new microwave resonant applicator will be developed to heat CFRTP, thermoplastic and metal filaments.

Typical microwave applicators for microwave heating are single or multi-mode (TE- or TM-mode) cavities or travelling wave applicators, the minimum size of which corresponds to about half the wavelength of the operation frequency [6-8]. At the widely used $2.45 \mathrm{GHz}$ ISM band, half wavelength in free space is $6.12 \mathrm{~cm}$, which is much bigger than typical filament diameters. A better approach to concentrate the microwave power to the targeted material is the use of coaxial applicators like an open-ended coaxial waveguide as used for the microwave drill [9] or coaxial antennas as used for materials heating [10] or tumor ablation [11]. Open-ended coaxial lines are also implemented for broadband dielectric spectroscopy in the microwave range $[12,13]$. Coaxial resonators have been widely researched to obtain low-loss filters [14] and Chebyshev low-pass filters [15] in wireless communication systems. A compact coaxial cavity resonator filter has been studied to operate at a frequency of $3 \mathrm{GHz}$ [16].

The above-mentioned research work and achievements prove the considerable efforts in the ongoing development of coaxial resonant cavity and filters. However, the emerging microwave heating assisted additive manufacturing (also known as 3D microwave printing or microwave additive manufacturing) of CFRTP structures brings new challenges to the design of an appropriate microwave applicator and has not been researched before. In this paper, the design principle and structure of a resonant coaxial applicator, and compact quarter wavelength filter are researched. By using finite element modelling, the scattering parameters of the applicator and filter have been simulated and compared with the measurement results.

\section{Design OF Small-size Coaxial Resonant ApPlicator}

The design of the small-size coaxial resonant applicator is based on a capacitive-loaded coaxial resonator. The detailed dimensions and 3D model are shown in Fig. 1. The outer 
diameter of the applicator is only $38 \mathrm{~mm}$ and the total length from inlet to outlet is about $150 \mathrm{~mm}$. The inner conductor has a diameter of $24 \mathrm{~mm}$ and connects the coupling pin of port 1 . The CFRTP, thermoplastic or metal filaments go through the inner conductor and are heated in the area between the two filters. A quarter wavelength impedance transformation coaxial line has been used to match the impedance of the standard $\mathrm{N}$ type connector and the coaxial cavity. The materials used to machine the applicator are listed in Fig. 1 (a). The filaments enter the applicator from the inlet (port 2) as shown in Fig. 1. (b) and the microwaves are coupled into the cavity through the port 1 to stimulate a TEM mode. Through involving the capacitive-loaded coaxial resonator, the heating area of the filaments is shrunk to a small area and the resonant frequency can easily be tuned by adjusting the length of the inner conductor. Different to hollow waveguides, coaxial waveguides do not have cutoff frequencies and therefore coaxial resonators can be designed at a very small size even at S-band (from 2 to $4 \mathrm{GHz}$ ). Since the generated TEM mode has an electric field direction, which is perpendicular to the filaments, this mode provides the highest penetration depth of the microwaves into the CFRTP filament. The simulation results of this applicator demonstrate that this designed structure has a resonant frequency at $2.45 \mathrm{GHz}$. Two compact quarter wavelength filters are installed in the applicator to limit the microwave heating area and to prevent microwave leakage (detailed information is discussed in section III). One filter is inserted in the inner conductor and another filter is integrated in the $6 \mathrm{~mm}$ diameter nozzle, as shown in Fig. 1 (a). The outlet of the nozzle is port 3 and a cooling fin has been machined to enhance the heat dissipation and improve the dimensional stability of the cavity. Furthermore, an infrared camera is applied to measure the filament heating temperature through the hole on the cavity, as illustrated in Fig. 1 (b).

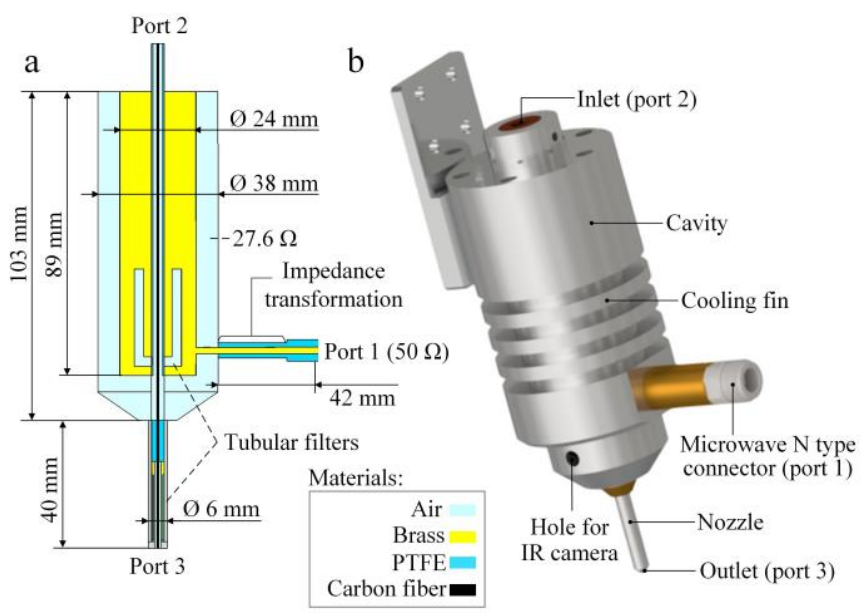

Fig. 1. (a) Schematic diagram and (b) 3D model of the coaxial resonant microwave applicator for microwave heating assisted additive manufacturing.

\section{Compact Tubular Filter and Equivalent Circuit}

Notch filters for coaxial transmission lines are often realized using parallel stubs, as shows in Fig. 2(a). However as those filters require contact to the center conductor they cannot be employed in the application of 3D printing process, where the center conductor is the moving CFRTP filament. The new tubular filter presented in this work is designed as a parallel RLC filter of $\lambda / 4$ length terminated by a short, as shows in Fig. 2(b). The direction of the electric field has been forced to turn 90 degrees and the $\lambda / 4$ length dielectric material transfers an electric wall (tangential electric field component is zero) to a magnetic wall. The tubular filter will start to work when using conductive filaments, and the filament plays the role as a part of the inner conductor. The designed filter requires only contact to the outer conductor and allows the filament to move freely. In addition, it enables the very compact form of the filter by aligning the stub coaxially to the transmission line and therefore provide the possibility to integrate the compact filter into the printing nozzle. The diameter does not influence the band-stop frequency of the tubular filter. Thus, it can be designed with an extremely compact size, e.g. with an outer diameter of only $4 \mathrm{~mm}$. The corresponding equivalent circuit using transmission lines and lumped elements are shown in Fig. 2(c) and (d). $P_{1}$ and $P_{2}$ are the ports of coaxial transmission lines. The series RLC circuit represents the coaxial line of the cavity, and the resistance $R_{0}$ and $R_{f}$ indicate the loss along conductive filaments. As shown in Fig. 2(d) of the equivalent circuit of the tubular filter, the filter is equivalent to a parallel RLC resonator, where $C_{f}$ is capacitive element and $L_{f}$ is inductive element in the filter. a

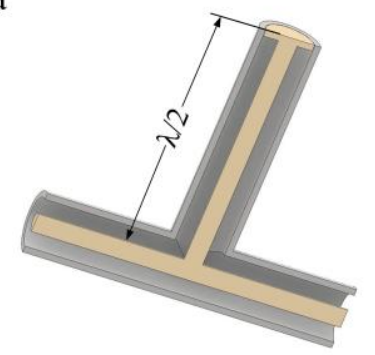

Parallel stub filter (only $1 / 2$ shown) b

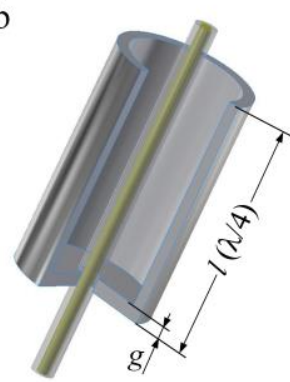

New tubular filter (only $3 / 4$ shown) c

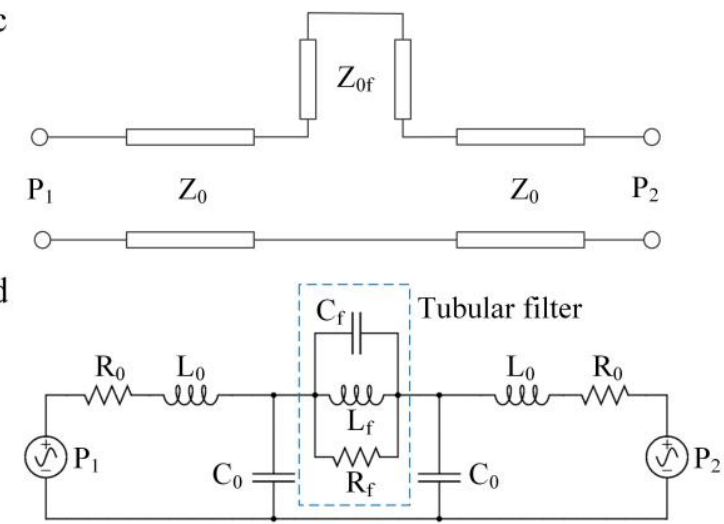

Fig. 2. Comparison of traditional notch filter (a) with developed compact tubular filter (b), (c) equivalent circuit 
using transmission lines, (d) equivalent circuit using lumped elements.

According to the transmission line theory [17], the input impedance $Z_{f}$ of the filter can be expressed as:

$Z_{f}=j Z_{0 f} \tan \beta l$

Where $Z_{0 f}$ is the line impedance of the tubular filter, $l$ is the length of the tubular filter, and $\beta$ is the propagation constant inside the filter. For the stub with a length of $\lambda / 4$, the input impedance will tend towards infinity and act as a perfect open. As can be seen from equation (1) the bandwidth of the filter depends mainly on the line impedance of the stub. In contrast to traditional parallel stubs where a low line impedance is required to achieve a low input impedance over a wide frequency band, this filter requires a high line impedance to increase the input impedance over a wider frequency band. Because the applicator is powered by a solid-state generator, the bandwidth and compact size of the filter can be balanced by tuning the output frequency of the generator. An equivalent circuit using lumped components can therefore be derived as shown in Fig. 2 (d). According to parallel RLC circuit approaches $[18,19]$, the circuit parameters can be expressed as the following equations:

$$
\begin{aligned}
& R_{f}=\left.2 Z_{0 f}\left(1 /\left|S_{21}\right|-1\right)\right|_{f=f_{c}} \\
& C_{f}=\frac{\sqrt{0.5\left(R_{f}+2 Z_{0 f}\right)^{2}-4 Z_{0 f}^{2}}}{2.83 \pi Z_{0 f} R_{f} \Delta f_{c}} \\
& L_{f}=\frac{1}{4\left(\pi f_{c}\right)^{2} C_{f}}
\end{aligned}
$$

The resistance of the filter is $R_{f}$, which depends on the property and diameter of the CFRTP filaments. $f_{c}$ is the resonant frequency of the filter, and $S_{21}$ is the transmission coefficient. $C_{f}$ is the capacitive reactance, and $L_{f}$ is the inductive reactance. $\Delta f_{c}$ is the $-3 \mathrm{~dB}$ bandwidth of $\mathrm{S}_{21}$.

\section{Electromagnetic Simulation AND CALCULATION}

To evaluate the scattering parameters $\left(S_{11}, S_{21}\right.$ and $\left.S_{31}\right)$ of the designed compact tubular filter and small-size applicator, the digital models have been established and calculated in a finite element modelling environment. The electrical properties of $\mathrm{T} 300$ carbon fiber ( $1 \mathrm{~K}$ and $6 \mathrm{~K}$, Toray Industries), polyamide (910 PA6, Taulamn3D) and polylactic acid (PLA, NatureWorks LLC) used in the simulation have been listed in Table 1. With regards to the conductive filaments, e.g. carbon fibers, the high resistance leads to Joule-heating in this domain rather than dielectric heating of thermoplastic materials under microwave radiation. As shown in Table 1, the reinforcement of carbon fibers not only improves the mechanical strength of printed parts but also reduce temperature gradients due to its high thermal conductivity.

First of all, the compact tubular filter is researched by using CST microwave studio, the electromagnetic field of the filter
TABLE I

ELECTRICAL PROPERTIES OF MATERIALS

\begin{tabular}{cccc}
\hline \hline Materials & $\begin{array}{c}\text { Electric } \\
\text { conductivity }\end{array}$ & Thermal conductivity & $\begin{array}{c}\text { Relative } \\
\text { permittivity }\end{array}$ \\
\hline Carbon fiber & $58824 \mathrm{~S} / \mathrm{m}$ & $10.5 \mathrm{~W} / \mathrm{m} \cdot \mathrm{K}$ & 1 \\
PA6 & $\sim 10^{-12} \mathrm{~S} / \mathrm{m}$ & $0.26 \mathrm{~W} / \mathrm{m} \cdot \mathrm{K}$ & $3.06[20]$ \\
PLA & $\sim 10^{-3} \mathrm{~S} / \mathrm{m}$ & $0.13 \mathrm{~W} / \mathrm{m} \cdot \mathrm{K}$ & $3.5[21]$ \\
PTFE & $5.1 \times 10^{-17} \mathrm{~S} / \mathrm{m}$ & $0.25 \mathrm{~W} / \mathrm{m} \cdot \mathrm{K}$ & 2.1 \\
\hline \hline
\end{tabular}

has been simulated and is shown in Fig. 3. The model of the microwave applicator's nozzle has been built and the tubular filter is inserted in the nozzle. The $6 \mathrm{~K}$ carbon fiber has an approx. $0.54 \mathrm{~mm}$ diameter and the PTFE material (blue area) has been applied to decrease the length of the filter, which is $21.5 \mathrm{~mm}$ as shown in Fig. 3 (a). The microwaves go inside the filter by taking the conductive filament as the inner conductor. Port 1 on the top of the nozzle is the input port of microwaves and port 2 is the outlet. It is evidence of how the tubular filter stopped the transmission of the microwaves, as shown in Fig. 3 (b) and (c). The electromagnetic energy is stored in the filter effectively and the outlet of the nozzle shows nearly zero field intensity. For the filter in the inner conductor of Fig. 1(a), the dielectric material is air and it has a length $l$ of $30.4 \mathrm{~mm}$. Both two filters have the same band-stop frequency at $2.45 \mathrm{GHz}$.

As demonstrated in Fig. 4, the influence of the filter length $l$ and gap thickness $g$ on the band-stop frequency have been researched. Through changing the length $l$ from 20.5 to 22.5 $\mathrm{mm}$, the frequency shifted from 2.66 to $2.43 \mathrm{GHz}$. On the other hand, the increasing of the gap thickness $g$ leads to a frequency variation from 2.34 to $2.43 \mathrm{GHz}$. Thus, the gap thickness, which decides by the parameter $C_{f}$ has a larger influence on the band-stop frequency compared with filter length.

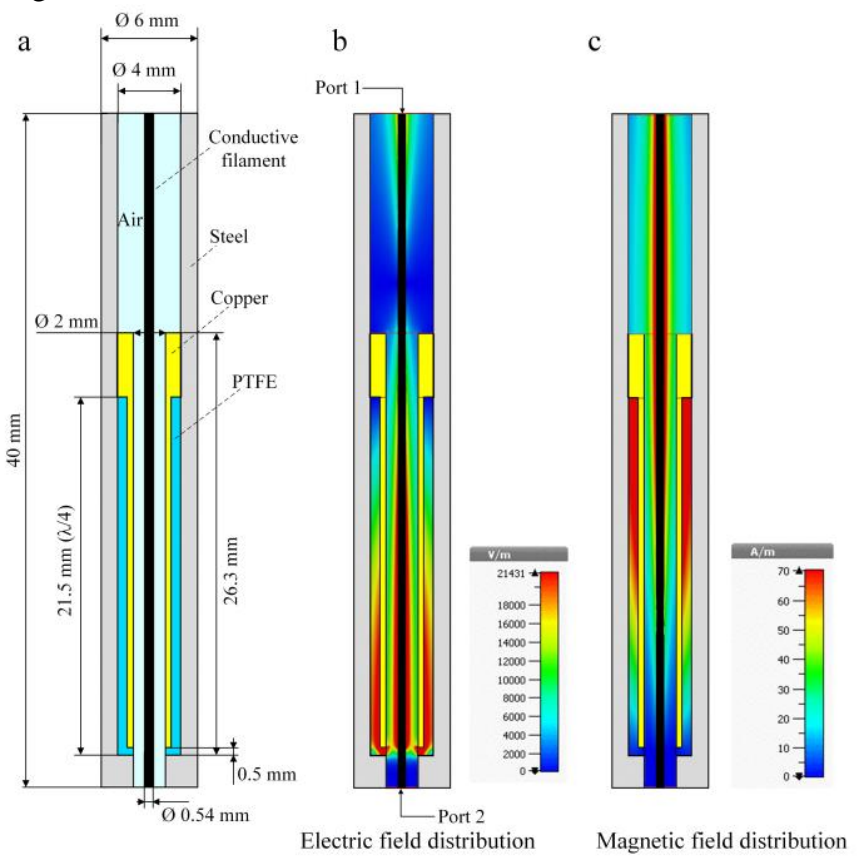

Fig. 3. (a) 3D model of the compact filter, simulated electric field (b) and magnetic field (c) distribution of the filter. 

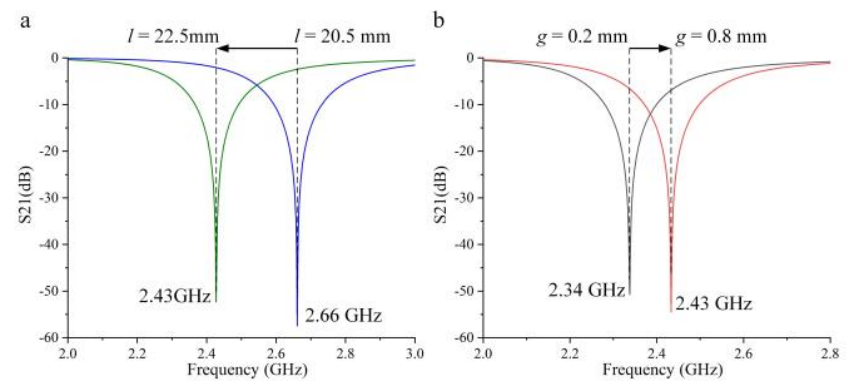

Fig. 4. Influence of the filter length $l$ (a) and gap thickness $g$ (b) to the band-stop frequency.

By using the compact tubular filter, the coaxial resonant microwave applicator is developed and the calculated electric fields with non-conductive and conductive filaments are shown in Fig. 5(a) and (b). When printing the non-conductive filament, such as thermoplastic materials, the heating spot locates on the resonant cavity, as shown in Fig. 5(a). In the case of applying CFRTP or metal filaments, the nozzle becomes part of the cavity and the two filters start to work. Due to the Joule-heating principle, the magnetic field dominates the heating spot of the conductive filament. As shown in Fig. 5(b), the heating spot for the conductive filament is inside the nozzle. By changing the length of the inner conductor made of brass (the length is $89 \mathrm{~mm}$ ) as shown in Fig. 1, the resonant frequency shifts from 2.4 to $2.5 \mathrm{GHz}$.

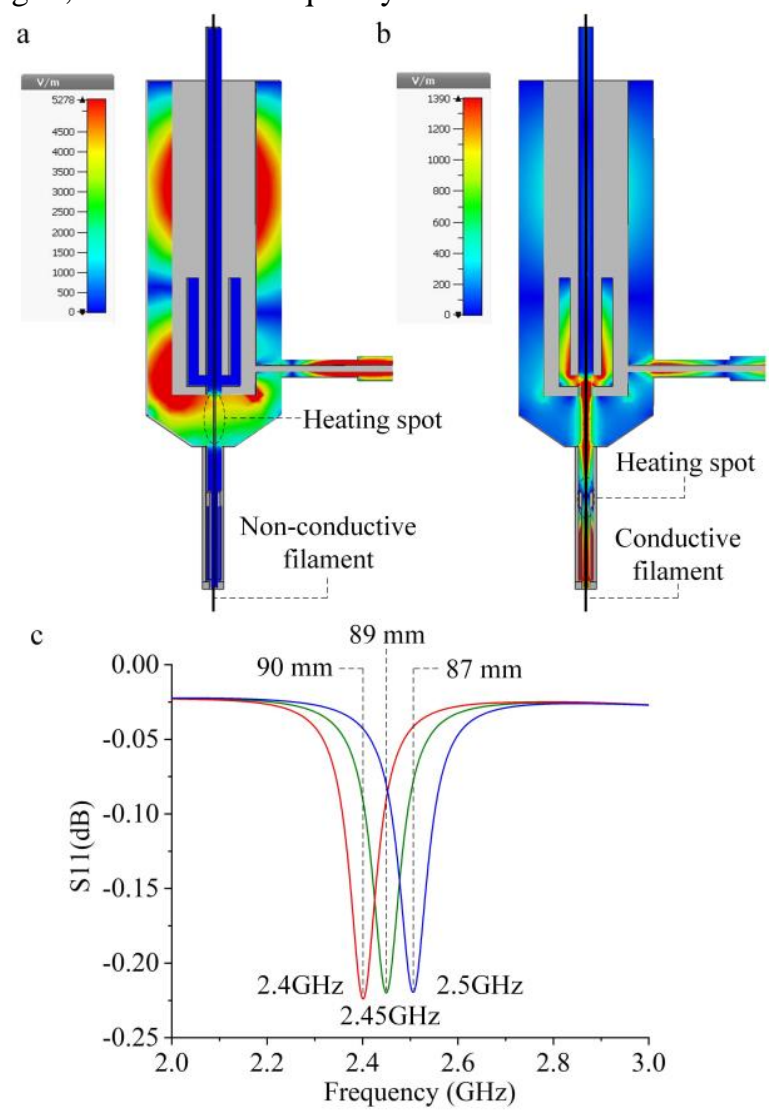

Fig. 5. Calculated electric fields of the developed applicator with non-conductive (a) and conductive (b) filaments, (c) tuning the resonant frequency of the applicator by changing the length of the inner conductor.

\section{EXPERIMENTAL VERIFICATION}

First, a compact tubular filter has been manufactured according to the $3 \mathrm{D}$ model and the $\mathrm{S}$ parameters have been tested under different conditions by using a two-port network analyzer (Rohde \& Schwarz, $9 \mathrm{kHz}-3 \mathrm{GHz}$ ), as shown in Fig. 6 . The whole structure of the filter is the same as the model shown in Fig. 3(a). The outer diameter of the steel tube is 6 $\mathrm{mm}$, and the inner diameter is $4 \mathrm{~mm}$. The copper and PTFE tubes have been combined to a quarter wavelength filter. The $6 \mathrm{~K}$ carbon fiber reinforced polyamide filament is plugged into two female SMA connectors and serves as the inner conductor.
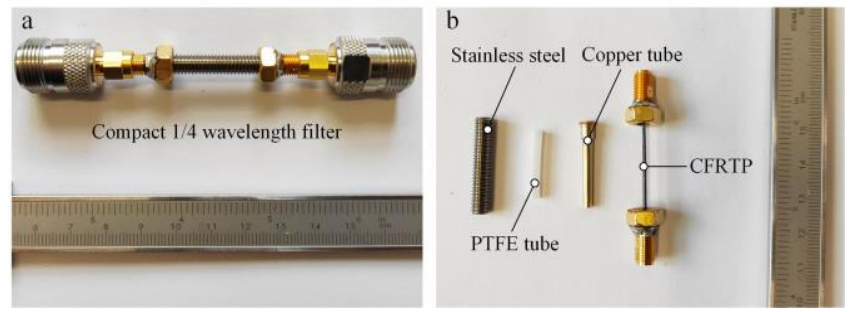

Fig. 6. (a) Compact tubular filter connected with SMA adapter, (b) exploded view of the filter.

The testing results for different parameters and filament materials are shown in Fig. 7. The compact quarter wavelength filter with a length of $l=21.5 \mathrm{~mm}$ and gap distance $g=0.5 \mathrm{~mm}$ shows a filtering ranges from 2.41 to 2.46 $\mathrm{GHz}(<-20 \mathrm{~dB})$, as represented in Fig. 7(a). Refer to the equations of equivalent circuit, the parameters of electrical components can be calculated. For the filter shown in Fig. 3, $Z_{0 f}=12 \Omega, R_{f}=1.18 \mathrm{k} \Omega, C_{f}=6.76 \mathrm{pF}, L_{f}=624.54 \mathrm{pH}$. By using the simulation tool of the equivalent circuit and input the above parameters, the $S_{21}$ curve of the filter is shown in Fig. 7(a). As the transmission line model assumes transmission in air, the length of the stub needs to be scaled according to the dielectric constant of PTFE. Comparison between the equivalent circuit and CST shows near perfect agreement. Furthermore, different kinds of materials have been tested in the experiments. As shown in Fig. 7(b), the CFRTP filaments have lower transmission coefficients than the copper wire. That means more microwave energy has been consumed when a CFRTP filament goes through the filter because carbon fibers can absorb more microwave power than copper materials. Furthermore, the $0.45 \mathrm{~mm}$ diameter filament exacerbates the impedance mismatch and increases the bandstop characteristics of the filter.
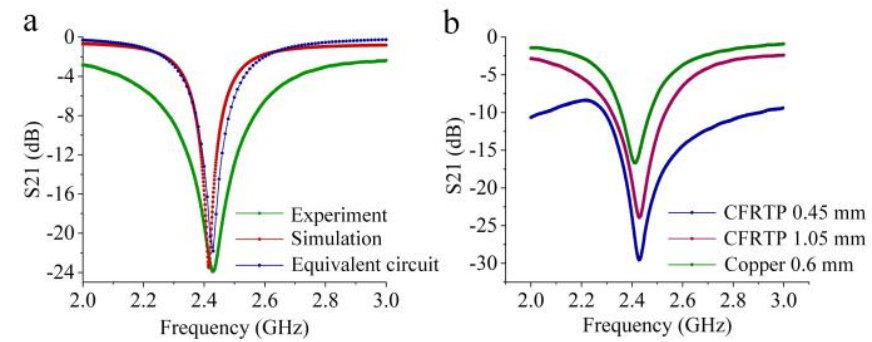

Fig. 7. Measurement and simulation results of the compact tubular filter, (a) comparison of experiment, simulation and 
circuit calculation results, (b) measured $S_{21}$ of different filaments.

The testing setup of the small-size coaxial resonant microwave applicator is shown in Fig. 8(a). The two-port network analyzer has been applied to measure the $S_{11}, S_{21}$ and $\mathrm{S}_{31}$ parameters. Port 1 connects to the microwave coupling port of the applicator and port 2 connects to the inlet. This setup allows measuring the $\mathrm{S}$ parameters of different filaments. First, a non-conductive PLA filament has been tested and compared with the simulation result. It can be seen that the simulation curve fits well with the experimental result, and $29 \%(-1.5 \mathrm{~dB})$ microwave energy has been coupled into the applicator, as shown in Fig. 8(b). Thus, the non-conductive thermoplastic filaments can be heated by using high power microwaves (detailed information described in Fig.9). As demonstrated in Fig. 8(c), by replacing the PLA filament with conductive copper filament, the resonant character of the applicator has been affected significantly. Because the two tubular filters have been activated and the microwave energy at the band-stop frequency, which is also the resonant frequency of the applicator, cannot leak to the ambient environment. Therefore, the curve of measured $S_{11}$ raises up between 2.4 to $2.6 \mathrm{GHz}$ and forms two peaks. Obviously, the frequencies of the two peaks lie outside of the stopping bandwidth of the filter and therefore, the microwaves are guided to the outside by the copper filament. Subsequently, a $1.05 \mathrm{~mm}$ diameter, 6K carbon fiber reinforced filament is plugged into an SMA adaptor and goes through the cavity. The measured $S_{11}, S_{21}$ and $S_{31}$ are shown in Fig. 8(d) to (f). It can be observed that about $37 \%$ microwave energy has been coupled into the applicator at $2.45 \mathrm{GHz}$ and most of the energy has been used to heat the CFRTP filament. The main goal to measure the $S_{21}$ parameter of the applicator is to evaluate the efficiency of the microwave filter. The $S_{21}$ parameter lower than $-20 \mathrm{~dB}$ from 2.42 to $2.53 \mathrm{GHz}$ indicates that the compact quarter wavelength filter is sufficiently broad-band and keeps the microwave energy inside the cavity at these frequencies. The dramatic difference between using the filter or not in the port 3 is shown in Fig. 8(f). By integrating the compact tubular filter inside the nozzle, the $S_{31}$ decreased from $-2.44 \mathrm{~dB}$ to $-15.78 \mathrm{~dB}$ at $2.45 \mathrm{GHz}$.

In order to characterize the heating performance of the small-size resonant microwave applicator, the temperature evolution during microwave heating at different power levels for different materials has been measured and is shown in Fig.9. A $300 \mathrm{~W}, 2.4-2.5 \mathrm{GHz}$ solid-state microwave generator (HBH Microwave Inc.) has been employed as a microwave source. When non-conductive filaments such as the polymer of PA6 and PLA have been tested in the microwave printing applicator, the behavior of the heating process is different to the CFRTP filament. As illustrated in Fig. 9(a), the static 1.75 $\mathrm{mm}$ diameter PA6 filament (dried in the oven for 24 hours) has been heated with a step-changing microwave power from 10 to $90 \mathrm{~W}$ every 10 seconds. When reaching temperatures beyond the glass transition temperature of the polymer, a hot spot is generated in the filament, which is located exactly in the heating area shown in Fig. 5(a) and that is also the measuring point of the infrared camera. This is due to a nonlinear increase of dielectric loss at a temperature beyond glass transition temperatures resulting in a thermal runaway, which is typical for thermoplastic materials [22].
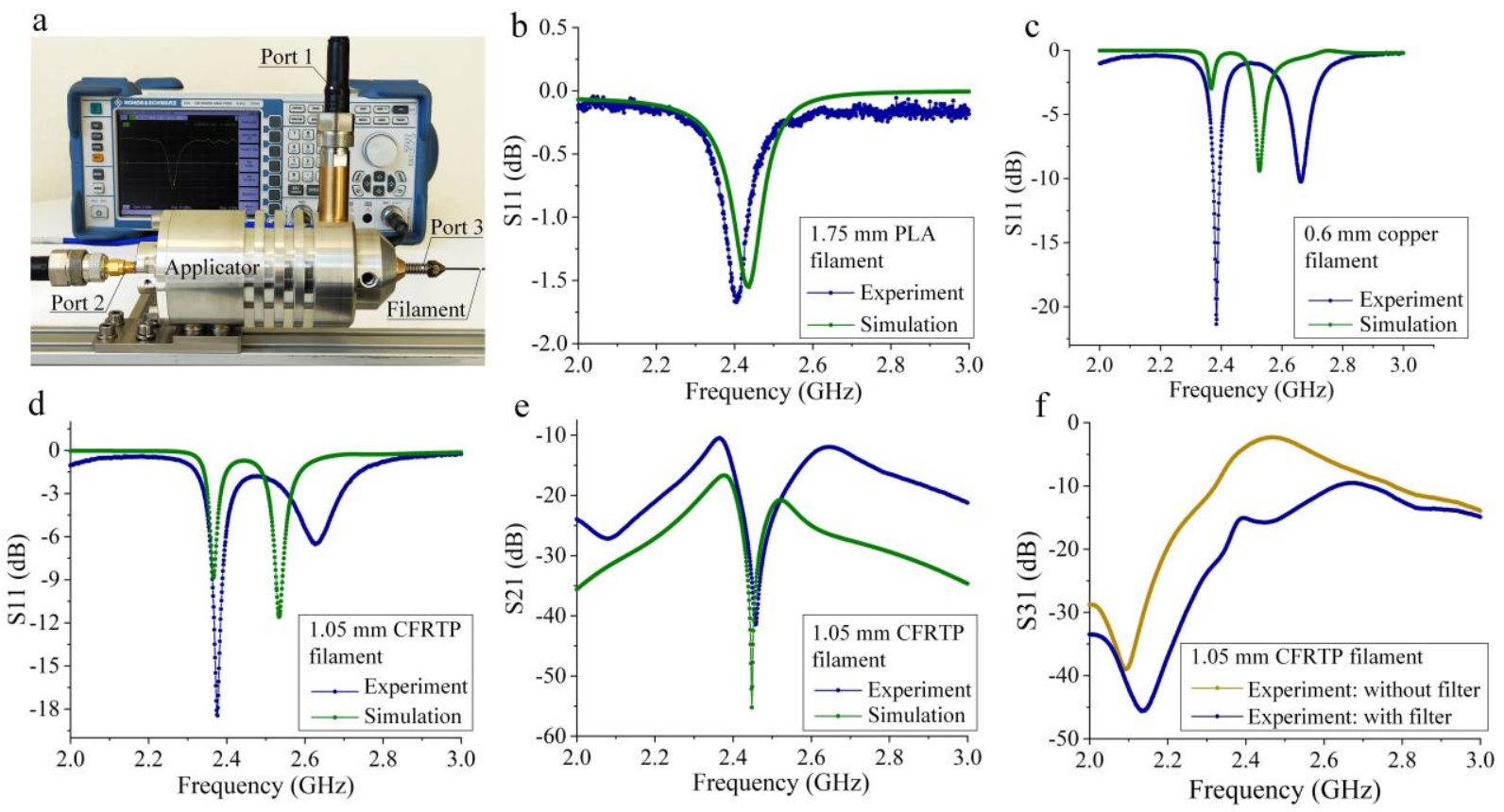

Fig. 8. (a) Testing setup of the single-mode microwave applicator, (b) measured $\mathrm{S}_{11}$ of the applicator with PLA filament, (c) measured $S_{11}$ of the applicator with copper filament inside, (d) to (f) measured $S_{11}, S_{21}$ and $S_{31}$ when using CFRTP filament. 

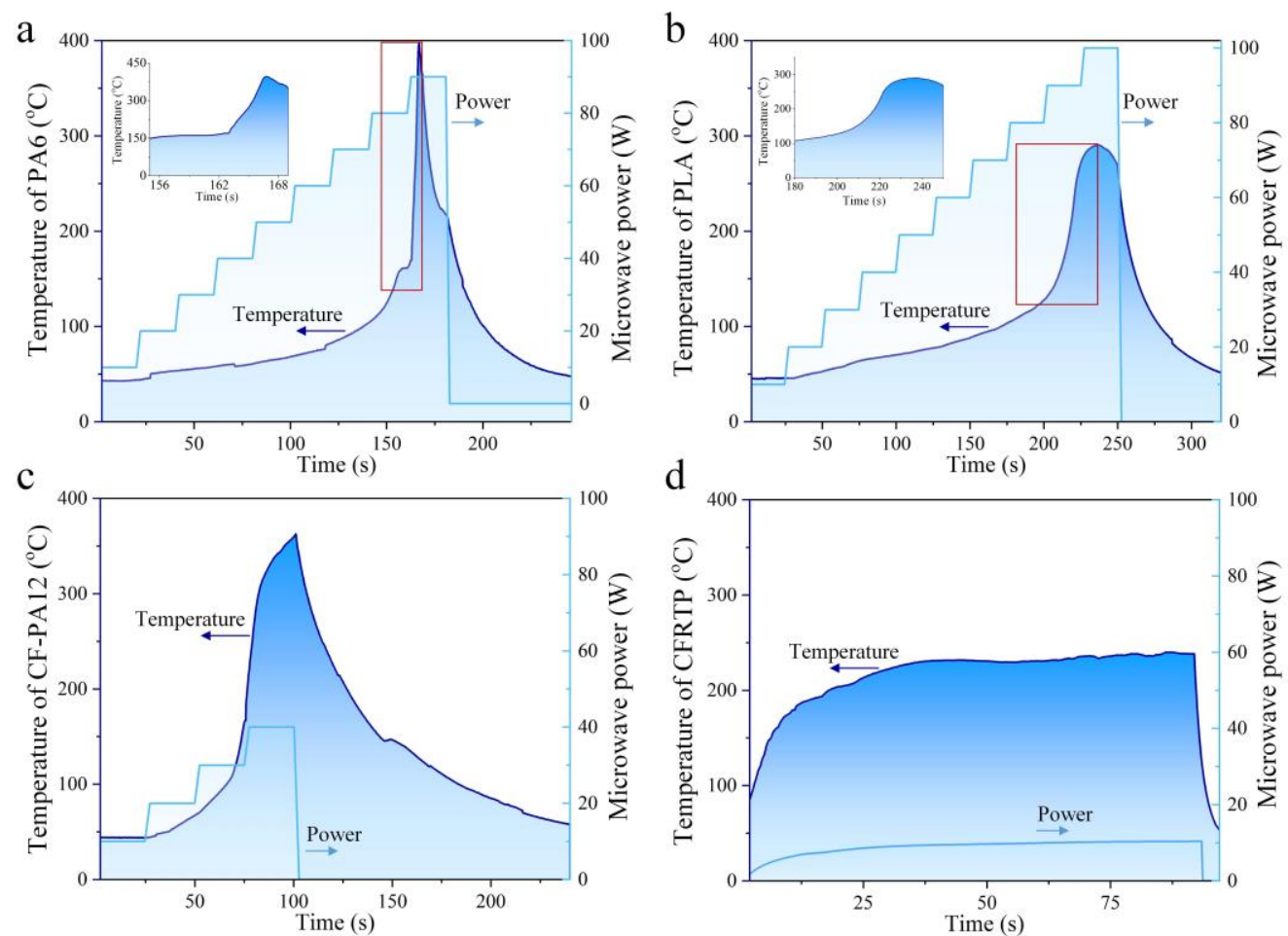

Fig. 9. Measured temperature evolution with varying microwave power for PA6 (a), PLA (b), CF-PA12 (c), and CFRTP filament (d) in the developed microwave applicator.

As shown in Fig. 9(a), the PA6 resin is slowly heated from room temperature. When the heating temperature arrives at $150^{\circ} \mathrm{C}$, the temperature explosively increases to $400^{\circ} \mathrm{C}$ in 4 seconds. The red box marked area has been zoomed in a small diagram at the left and indicates how fast the temperatures can change. After that, the PA6 filament separates into two melting drops and the temperature decreases. For the PLA resin, the microwave temperature has almost the same correlation with microwave power. The difference is that the PLA resin has a lower loss tangent than the PA6 resin, as shown in Table 1. Thus, the response to microwave power is much blunter.

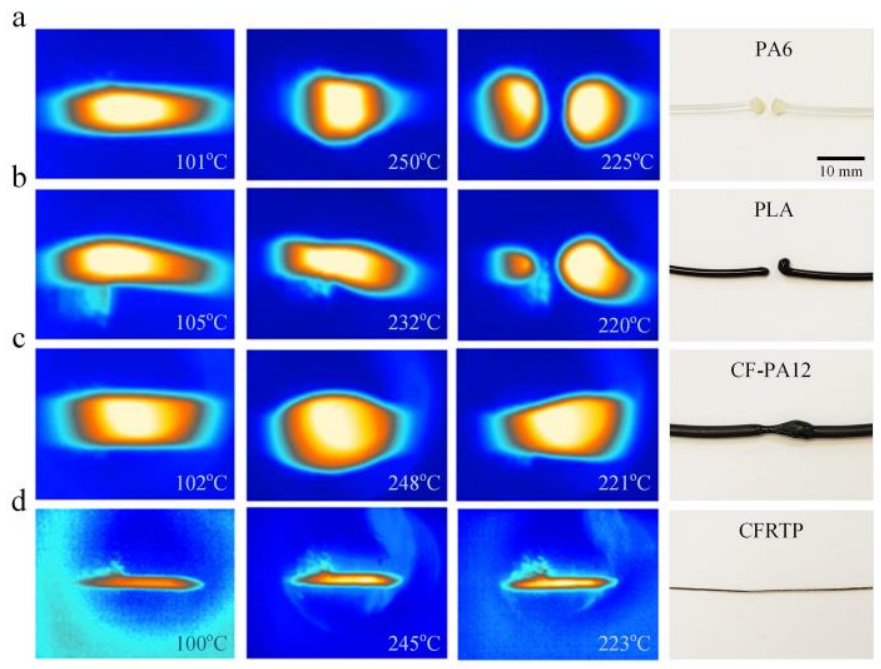

Fig. 10. Infrared images of the microwave heated filaments at different stages, (a) PA6, (b) PLA, (c) CF-PA12, (d) CFRTP.
By mixing with chopped carbon fibers, the PA12 resin shows different microwave heating behavior, as shown in Fig.9(c). Around $20 \mathrm{~W}$ power can heat the CF-PA12 filament (15\% fiber volume content of chopped carbon fibers, Fillamentum Industrial) to $200^{\circ} \mathrm{C}$ and obviously, the carbon fiber is in the domain to transfer the microwave energy into thermal heat. The filament has been heated quickly and the additional strength provided by chopped carbon fibers also prevents the filament from melting to drops (more information can be found in Fig.10). In Fig. 9(d), a static $0.45 \mathrm{~mm}$ CFRTP filament has been tested in the microwave applicator with the temperature controlled by a PID controller. Compared with all other filaments, the CFRTP allows a much higher heating rate and efficiency. The filament can be heated up to $250^{\circ} \mathrm{C}$ with $10 \mathrm{~W}$ microwave power only and a constant temperature can be maintained. The infrared images of the four kinds of filaments have been recorded and are shown in Fig. 10. The maximum temperatures captured in these images are marked on the right corner and the figure of the samples share the same size bar. In Fig. 10(a) and (b), at the melting temperature, the pure plastic filaments melt into drops and fall down to the printing bed. The chopped carbon fiber reinforced PA12 has a large diameter and changes its shape when melted as shown in Fig. 10(c). Due to the small diameter of the CFRTP filament, the hot spot demonstrates a thinner shape. The relatively fixed profile of the filament also ensures that during the high-speed microwave printing process, the temperature measurement is more precise and in favor of improving printing quality. 


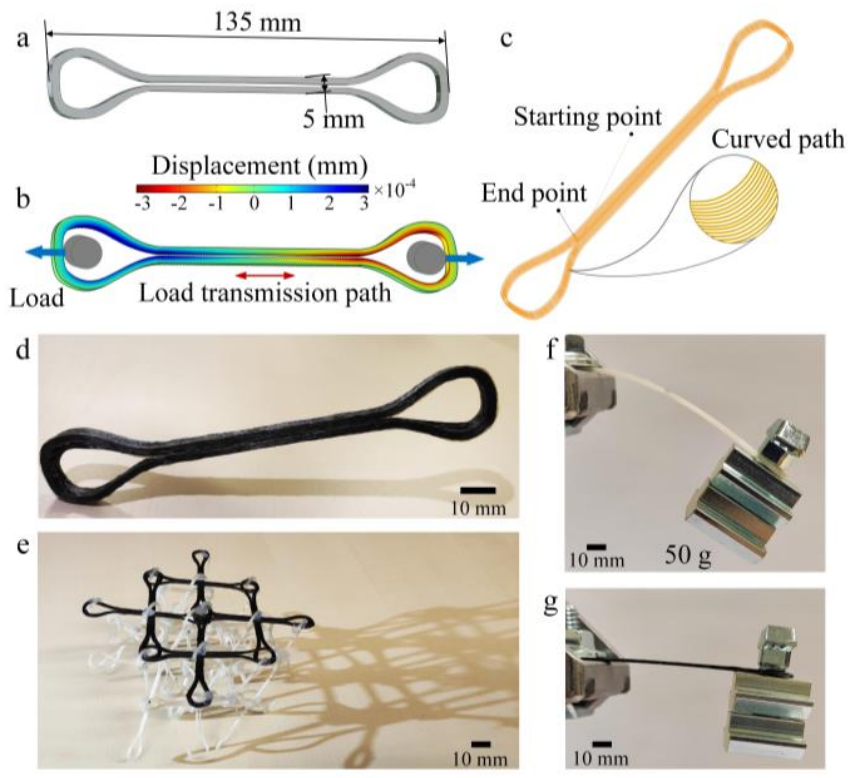

Fig. 11. (a) Dimension of the lightweight CFRTP connector, (b) mechanical analysis under tensile load, (c) planned printing path, (d) printed CFRTP connector, (e) assembly of a complex spatial lattice using printed connectors, and bending resistance of (f) PLA connector and (g) CFRTP connector.

To validate the developed coaxial resonant microwave applicator, a CFRTP part is printed in this paper. Connectors have been widely used to connect components and bear tensile loads. Usually, metallic parts are heavy and break easily. Continuous carbon fiber reinforced thermoplastic connector can provide higher performance even at a small scale, as shown in Figure 11(a). Lightweight CFRTP connectors manufactured using the developed microwave applicator can print the continuous fibers along the load transmission path and significantly increase the strength to weight ratio. The load applied on two bolts at the connection areas will generate stresses in the longitudinal direction inside the straight beam elements (simulated in COMSOL and the homogenous material properties of PLA have been used), as shown in Figure 11(b). Thus, a contour path has been planned to print the lightweight connector with continuous fibers reinforced in the direction of the stress vectors, as shown in Figure 11(c). The printing speed of this part is $35 \mathrm{~mm} / \mathrm{s}$ and much higher than the current maximum $10 \mathrm{~mm} / \mathrm{s}$ printing speed of CFRTP. The printed continuous carbon fiber reinforced polyamide part demonstrated higher bending strength than a PLA connector with the same geometry and dimension, as illustrated in Figure 11(f) and 11(g). The printed large size connector and a complex spatial lattice assembled using small CFRTP connectors (printed with $1 / 2$ scale of the original shape) are shown in Figure 11(d) and 11(e).

\section{CONCLUSION}

Microwave additive manufacturing is an emerging 3D printing technology, which can provide higher printing speed of multiple materials, e.g. continuous carbon fiber reinforce plastics, thermoplastics and metals, as compared to state-ofthe-art printing methods. Furthermore, due to volumetric heating, the printing dimension is not limited to small filament diameters. In this paper, the coaxial resonant microwave applicator with two compact quarter wavelength filters has been developed and can effectively heat non-conductive and conductive filaments. For a small heating spot of the CFRTP filament, about $37 \%$ of microwave energy has been absorbed and transferred into heat at $2.45 \mathrm{GHz}$. The compact filter with a $4 \mathrm{~mm}$ outer diameter shows a broad-band filtering property $(<-20 \mathrm{~dB})$ ranges from 2.41 to $2.46 \mathrm{GHz}$, and the equivalent circuit has been established to analyze the influence of structure parameter on the resonant frequency of the filter. The heating behavior of the CFRTP filament indicates that by using $10 \mathrm{~W}$ power, the temperature can raise up to $250^{\circ} \mathrm{C}$. This robust and tunable applicator allows the rapid heating of multiple materials, especially the high-performance continuous carbon fiber reinforced plastics with different diameters. In future, the size of the applicator could be reduced furthermore with improved coupling efficiency. The applicators will be installed on a multi-robot printing system and it will be applied to manufacture complex spatial lightweight structures, which could contribute substantial benefits for the reduction of $\mathrm{CO}_{2}$ emission by less weight and materials for vehicles.

\section{REFERENCES}

[1] Li, N., Link, G., Jelonnek, J. (2020). Rapid 3D microwave printing of continuous carbon fiber reinforced plastics. CIRP Annals. 69(1), 221-224.

[2] Tian, X., Liu, T., Yang, C., Wang, Q., Li, D. (2016). Interface and performance of 3D printed continuous carbon fiber reinforced PLA composites. Composites Part A: Applied Science and Manufacturing, 88, 198-205.

[3] Li, N., Li, Y., Liu, S. (2016). Rapid prototyping of continuous carbon fiber reinforced polylactic acid composites by 3D printing. Journal of Materials Processing Technology, 238, 218-225.

[4] L. G. Blok, M. L. Longana, H. Yu, and B. K. S. Woods, "An investigation into 3D printing of fibre reinforced thermoplastic composites," Additive Manufacturing, vol. 22, pp. 176-186, Aug. 2018.

[5]Li, N., Link, G., Jelonnek, J. (2020). Production of continuous carbon fiber reinforced polyamide filaments for microwave additive manufacturing. SAMPE Europe Conference 2020 Amsterdam.

[6]Folli, A., Choi, H., Barter, M., Harari, J., Richards, E., Slocombe, D., Porch, A., Murphy, D. M. (2020). A novel dual mode X-band EPR resonator for rapid in situ microwave heating. Journal of Magnetic Resonance, 310, 106644

[7] Alekseev, V. S., Zakharov, V. V., Artyukhov, I. I., Trigorly, S. V. (2019, October). Numerical Simulation of the Processes of Microwave Heating of Dielectrics in Traveling Wave Chambers. In 2019 International Conference on Electrotechnical Complexes and Systems (ICOECS) (pp. 1-4). IEEE.

[8] Bae, S. H., Jeong, M. G., Kim, J. H., Lee, W. S. (2017). A continuous power-controlled microwave belt drier improving heating uniformity. IEEE Microwave and wireless components letters, 27(5), 527-529.

[9] Meir, Y., Jerby, E. (2012). Localized rapid heating by low-power solidstate microwave drill. IEEE transactions on microwave theory and techniques, 60(8), 2665-2672.

[10] Yang, D., Bertram, J. M., Converse, M. C., O'Rourke, A. P., Webster, J. G., Hagness, S. C., Will, J.A., Mahvi, D. M. (2006). A floating sleeve antenna yields localized hepatic microwave ablation. IEEE transactions on biomedical engineering, 53(3), 533-537.

[11] Gentili, G. B., Linari, M., Longo, I., Ricci, A. S. (2009). A coaxial microwave applicator for direct heating of liquids filling chemical 
reactors. IEEE transactions on microwave theory and techniques, 57(9), 2268-2275.

[12] La Gioia, A., Porter, E., Merunka, I., Shahzad, A., Salahuddin, S., Jones, M., O'Halloran, M. (2018). Open-ended coaxial probe technique for dielectric measurement of biological tissues: Challenges and common practices. Diagnostics, 8(2), 40.

[13] Hagl, D. M., Popovic, D., Hagness, S. C., Booske, J. H., Okoniewski, M. (2003). Sensing volume of open-ended coaxial probes for dielectric characterization of breast tissue at microwave frequencies. IEEE Transactions on Microwave Theory and Techniques, 51(4), 1194-1206.

[14] Li, H. Y., Xu, J. X., Zhang, X. Y., Zhao, X. L. (2018). Low-loss narrowband filtering switch based on coaxial resonators. IEEE Access, 6, 47717-47722.

[15] Zhang, Z. C., Yang, J. P., Li, Q. Y., Lv, J. X. (2019, May). Design of Miniaturized Bandpass Filter Using Modified Coaxial Cavity Resonator. In 2019 International Conference on Microwave and Millimeter Wave Technology (ICMMT) (pp. 1-3). IEEE.

[16] Salek, M., Shang, X., Lancaster, M. J. (2019). Compact S-Band Coaxial Cavity Resonator Filter Fabricated By 3-D Printing. IEEE Microwave and Wireless Components Letters, 29(6), 382-384.

[17] Pozar D.M., Microwave Engineering. 2nd ed. John Wiley \& Sons, Inc., 1997

[18] Aykan, A. (1998). Calibration of circular loop antennas. IEEE Transactions on Instrumentation and Measurement, 47(2), 446-452.

[19] Angkawisittpan, N. (2012). Miniaturization of bandstop filter using double spurlines and double stubs. Przegląd Elektrotechniczny (Electrical Rev, 11, 178-81.

[20] Reyes, N., Casado, F., Tapia, V., Jarufe, C., Finger, R., Bronfman, L. (2018). Complex dielectric permittivity of engineering and 3D-printing polymers at Q-band. Journal of Infrared, Millimeter, and Terahertz Waves, 39(11), 1140-1147.

[21] Huber, E., Mirzaee, M., Bjorgaard, J., Hoyack, M., Noghanian, S., Chang, I. (2016, May). Dielectric property measurement of PLA. In 2016 IEEE International Conference on Electro Information Technology (EIT) (pp. 0788-0792). IEEE.

[22] Lukasheva, N. V., Tolmachev, D. A., Nazarychev, V. M., Kenny, J. M., Lyulin, S. V. (2017). Influence of specific intermolecular interactions on the thermal and dielectric properties of bulk polymers: atomistic molecular dynamics simulations of Nylon 6. Soft Matter, 13(2), 474-485.

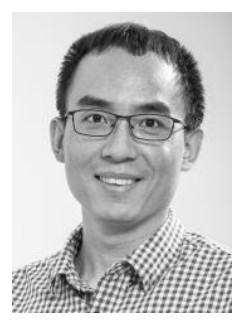

Nanya $\mathrm{Li}$ received the B.Sc. and Ph.D. degrees in aerospace manufacturing engineering from Nanjing University of Aeronautics and Astronautics, Nanjing, China, in 2010 and 2017, respectively. From 2018 to 2020, he was an Alexander von Humboldt research fellow with the Institut für Hochleistungsimpuls- und Mikrowellentechnik (IHM), Karlsruhe Institute of Technology (KIT), Germany. Since October 2020, he becomes the KIT Young Investigator Group Preparation Program (YIG Prep Pro) funded fellow. He has published 26 peerreviewed papers, granted 26 Chinese patents, published 1 German patent, and 1 international patent covering the 3D microwave printing technologies, prototype and microwave curing methods of continuous carbon fiber reinforced polymer composites. He developed the first 3D microwave printing system for continuous carbon fiber reinforced thermoplastics, which is much faster than traditional printers. Due to his achievements, he won the $1^{\text {st }}$ prize of KIT "NEULAND" innovation award 2020, the finalist of JEC World innovation award 2020. He also won the $1^{\text {st }}$ prize of the " 1 st MIIT Innovation and Entrepreneurship Award 2019” organized by MIIT of China in 2019.

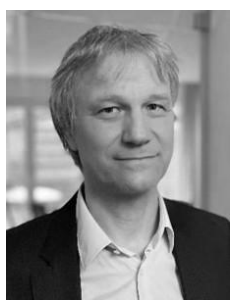

Guido Link received the Dipl.-Phys. and Dr. rer. nat. degree in physics from the Technical University Karlsruhe, Germany in 1990 and 1993, respectively. His diploma thesis and graduate research was devoted to the frequency and temperature dependent dielectric characterization of low loss ceramics and ionic crystals. Since 1993, he has been working at the Karlsruhe Institute of Technology, Germany (former Forschungszentrum Karlsruhe) in the field of high power microwave and millimeter-wave processing of materials as a team leader at the Institute for Pulsed Power and Microwave Technology. His research interest includes dielectric measurements, design and simulation of microwave systems and processes, microwave assisted sintering, curing of polymer composites, additive manufacturing, plasma chemistry. He authored or coauthored more than 200 contributed papers to international scientific conference and more than 30 papers in scientific journal and holds 8 patents.

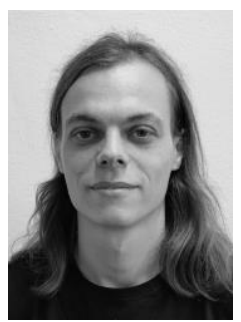

Moritz Engler received the B.Sc. and M.Sc. degrees in electrical engineering from Karlsruhe Institute of Technology (KIT), Karlsruhe, Germany, in 2016 and 2018 respectively. He is currently a researcher with the Institute for Pulsed Power and Microwave Technology (IHM) at KIT, where his research is in microwave processing and characterization of composite materials.

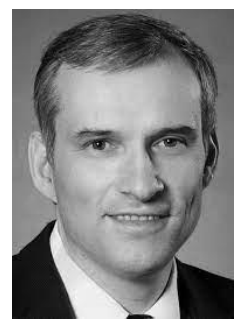

John Jelonnek received the Dipl.-Ing. and Dr.-Ing. degrees in electrical engineering from Hamburg University of Technology (TUHH), Germany, in 1991 and 2000, respectively. At TUHH he developed rigorous self-consistent analyses for gyrotron oscillators with particular focus on rigorous timedomain simulation at mismatched conditions and injection locking. From 1997 to 2011, John Jelonnek was working in several different positions in industry. Since 2011, John Jelonnek is the Director of the Institute for Pulsed Power and Microwave Technology (IHM) at Karlsruhe Institute of Technology (KIT), Germany. Both, research and development of high-power microwave sources, with particular focus on megawatt-class gyrotrons, and applications of microwaves to energy efficient industrial processes using dielectric heating and microwave plasmas are in the focus. John Jelonnek is a Professor for high-power microwave technologies at KIT. 